Loading

The Journal of the Canadian Game Studies Association

Collectionner des jeux vidéo au Québec Une communauté naviguant entre société distincte et mondialisation incontournable

Collecting Video Games in Québec A Community Navigating between Distinct Society and Forced Globalization

\title{
Adam Lefloïc-Lebel
}

Volume 14, Number 23, 2021

Le jeu vidéo au Québec

The Video Game in Quebec

URI: https://id.erudit.org/iderudit/1078731ar

DOI: https://doi.org/10.7202/1078731ar

See table of contents

Publisher(s)

Canadian Game Studies Association

ISSN

1923-2691 (digital)

Explore this journal

Cite this article

Lefloïc-Lebel, A. (2021). Collectionner des jeux vidéo au Québec : une communauté naviguant entre société distincte et mondialisation

incontournable. Loading, 14(23), 115-132. https://doi.org/10.7202/1078731ar
Article abstract

This article takes a look at the community of game collectors in Quebec, first by exploring how ludovideophily compares itself from classic collecting. We then isolate Quebec video game collection to identify how the community navigates through a world that is even more open and that interacts mostly in English. Do specifically Quebec centric attributes exist? An incursion inside this group will highlight our reflection and help confirm if these collectors meld themselves in the bigger group or if they stick their head high enough to differentiate from the international communities.
Copyright (c) Adam Lefloïc-Lebel, 2021

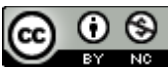

This document is protected by copyright law. Use of the services of Érudit (including reproduction) is subject to its terms and conditions, which can be viewed online.

https://apropos.erudit.org/en/users/policy-on-use/ 


\title{
Collectionner des jeux vidéo au Québec : une communauté naviguant entre société distincte et mondialisation incontournable
}

\section{Collecting Video Games in Québec : A Community Navigating between Distinct Society and Forced Globalization}

\author{
Adam Lefloïc Lebel \\ Université du Québec en Abitibi-Témiscamingue \\ lefa08@uqat.ca \\ Résumé
}

Cet article pose un regard sur la communauté de collectionneurs vidéoludiques du Québec, d'abord en clarifiant comment la ludovidéophilie se compare aux autres mouvements de collection plus classiques. Ensuite, en isolant le collectionnement de jeux vidéo au Québec, nous chercherons à voir comment cette communauté évolue à travers un monde de plus en plus ouvert et qui interagit majoritairement en anglais. Existe-t-il des traits de ludovidéophilie qui soient spécifiquement québécois ? Une incursion parmi cette communauté éclairera notre réflexion et confirmera s'il s'agit vraiment d'une communauté de «petits Gaulois dans une mer d'Anglais » ou bien si ses membres réussissent aisément à tirer leur épingle du jeu (vidéo).

\section{Mots-clés}

Jeu vidéo, collection, collectionneur, Québec.

\begin{abstract}
This article takes a look at the community of game collectors in Quebec, first by exploring how ludovideophily compares itself from classic collecting. We then isolate Quebec video game collection to identify how the community navigates through a world that is even more open and that interacts mostly in English. Do specifically Quebec centric attributes exist? An incursion inside this group will highlight our reflection and help confirm if these collectors meld themselves in the bigger group or if they stick their head high enough to differentiate from the international communities.
\end{abstract}

\section{Keywords}


Video game, collector, collection, Quebec.

\section{Appuyez sur START pour commencer}

Quand on pense au jeu vidéo au Québec, c'est immédiatement son industrie qui nous vient à l'esprit. Avec de gros studios comme Ubisoft, Warner Bros., SquareEnix, ainsi que la Guilde du jeu vidéo au Québec, la Belle Province est aujourd'hui le lieu d'une florissante production. Il n'en demeure pas moins que, comme phénomène culturel, le jeu vidéo repose aussi sur les joueurs et leurs pratiques. Ces derniers ne font pas que jouer ; ils critiquent, créent du contenu jouable, maîtrisent complètement leurs jeux favoris et font partager leur expérience à travers des réseaux qui rassemblent une communauté élargie de joueuses et de joueurs. L'une de ces pratiques est trop souvent oubliée : le collectionnement de jeux. Des joueurs nostalgiques se procurent et accumulent effectivement les plateformes ainsi que les jeux de leur enfance - parfois plus vieux qu'eux - et les exposent même dans des pièces dédiées à leurs trésors.

Avec une collection comptant plus de 800 jeux, consoles et artefacts, je fais moi-même partie de cette communauté de collectionneurs. C'est d'abord ce qui m'a amené à poursuivre des études supérieures en études vidéoludiques et à participer à plusieurs événements, colloques et conférences en tant qu'organisateur, conférencier ou spectateur. Depuis, je m'interroge sur la présence ou non de caractéristiques distinctes chez mes comparses québécois compte tenu de l'importance du marché du jeu vidéo dans la province. Le Québec ayant réussi à tirer son épingle du jeu et à se démarquer dans cette industrie hautement compétitive, il faut se demander si cela a eu des répercussions sur les amateurs absorbés dans un passe-temps en constante évolution. C'est pourquoi cet article se penche sur le collectionnement et plus spécialement sur les regroupements de collectionneurs de jeux vidéo au Québec. Il s'agit de décrire leurs habitudes et de les comparer avec celles de leurs confrères et consœurs à l'extérieur de la province.

\section{Qu'est-ce qu'un collectionneur et une collection?}

Ma recherche veut répondre à la question suivante : Qu'est-ce que collectionner le jeu vidéo au Québec ? Il m'apparait pertinent de comprendre d'abord le collectionneur dans son sens large avant de resserrer la perspective sur le jeu vidéo. Qu'ils accumulent des cuillères à café, des cartes de hockey ou des jeux vidéo, les collectionneurs sont de véritables passionnés dont les comportements, très semblables, ont intéressé plusieurs chercheurs déjà.

Cette fascination pour la collection n'émane pas seulement de notre culture de consommation contemporaine. Jean Baudrillard s'y est intéressé dans Le système des objets (1978), où il mène une réflexion sur l'objet et notre rapport à l'objet. Son concept d'objet ancien ouvre le champ à la collection. Pour Baudrillard, cet objet est « purement mythologique dans sa référence au passé. Il n'a plus d'incidence pratique, il est là uniquement pour signifier » $(1978$, p. 104). On reconnait dans cette courte description l'objet de collection qui n'est plus utilisé par son propriétaire, par exemple un jeu qui se retrouve sur un présentoir plutôt que dans une console où il est prêt à être joué. On note donc que l'objet prend son sens une fois qu'il s'ajoute au reste de la collection: « c'est moins le livre [ou le jeu] qui compte que l'instant où il est rangé près des autres sur le rayon de la bibliothèque » (Baudrillard, 1978, p. 147). 
Dans un projet inachevé de Walter Benjamin, The Arcade Project, on trouve plusieurs notes portant directement sur le collectionnement, la collection et le collectionneur. Ce dernier y est décrit comme un personnage tactile et avare de son savoir, qui se concentre sur la possession d'objets physiques qu'il accumule comme une collection de connaissances dans un domaine pointu (Benjamin et Tiedemann, 2002, p. 206). Comme chez Baudrillard, il est précisé que les acquisitions sont détachées de leurs fonctions habituelles. Il suffit de posséder l'objet et de le disposer dans une série bien précise : « The collector [...] brings together what belongs together » (ibid.). Ainsi, un nouvel objet offre beaucoup plus au collectionneur que sa simple utilisation classique : l'acquisition représente un pas de plus dans la série à compléter et, surtout, une nouvelle source de connaissance venant bonifier sa propre encyclopédie.

Russel W. Belk a plus récemment étudié en profondeur la collection et les collectionneurs. Dans Collecting in a Consumer Society, il se penche sur l'histoire et l'évolution du collectionnement de la Grèce antique jusqu'à aujourd'hui. Ses travaux le conduisent à une définition claire et concise du phénomène : « the process of actively, selectively, and passionately acquiring and possessing things removed from ordinary use and perceived as part of a set of non-identical objects or experiences » (Belk, 1995, p. 67). On y retrouve déjà des concepts clés qui semblent de plus en plus inévitables lorsque l'on parle de collection, comme la notion de sérialisation et l'idée que les objets acquis ne sont pas utilisés. Belk consacre un chapitre au collectionneur privé et se concentre sur ses comportements et ses rapports avec la communauté de collectionneurs autour de laquelle il gravite.

Ces concepts clés que sont la sérialisation et l'utilisation ont été approfondis par Catherine Carrey dans son article « Modeling Collecting Behavior: The Role of Set Completion » (2008). Dans son évaluation des comportements de collectionneurs, elle propose un troisième concept, lié à la valeur sociale de l'objet. Contrairement à un consommateur « ordinaire » qui valorise l'acquisition d'un objet pour son utilité principale, le collectionneur lui accorde en plus une valeur pour sa contribution à l'ensemble de la collection (Carrey, 2008, p. 340). L'addition à la collection représente en quelque sorte un ajout au statut social du collectionneur, à son estime, à l'accomplissement de sa collection, ou encore un symbole de ses connaissances sur le sujet (Carrey, 2008, p. 339). Ces concepts complètent bien, à mon avis, les définitions du collectionneur et sont particulièrement applicables au collectionnement de jeux vidéo.

Enfin, dans La psychologie du collectionneur, Hubert Van Gijseghem caractérise le collectionneur à partir d'entretiens qu'il a catalogués et analysés efficacement. À travers une typologie basée sur le type d'objet collectionné, Gijseghem propose une définition du collectionneur qui englobe ce qui a été fait avant lui, dont les auteurs cités précédemment. ${ }^{1}$ Cette définition m'apparaissant comme la plus complète description du phénomène, c'est sur elle que s'appuient mes travaux et le présent article. La voici donc (je souligne).

Le collectionneur est aux prises avec une passion précise : la pulsion sinon la compulsion d'établir à partir d'un premier objet investi une série de semblables -mais différents - appartenant à la même catégorie d'objets. Il trouve du plaisir à chercher, à trouver, à acquérir, à organiser, à cataloguer, à entreposer, à regarder, à exhiber (ou à cacher) ses objets. Son but n'est ni le commerce ni l'investissement. Il établit un lien d'une grande intimité avec ses objets et, de ce fait, il ne peut que très difficilement s'en 
défaire. L'objet convoité n'a pas ou n'a plus d'utilité fonctionnelle et, ainsi, est considéré par le non-collectionneur comme «inutile», puisqu'il ne sert pas. La passion des collectionneurs se porte habituellement sur plusieurs catégories d'objets en même temps, ce qui en fait des « polycollectionneurs ».

Hubert Van Gijseghem, p. 207 (2014). ${ }^{2}$

Plus près du monde vidéoludique, Toivonen et Sotamaa abordent la collection en s'intéressant aux joueurs et à leur intérêt pour ces jeux qui se présentent sur des supports tangibles plutôt que dématérialisés. L'attrait de la collection serait selon eux intrinsèque au jeu vidéo puisqu'il se manifeste dans la mécanique même du jeu, au sens large où dans plusieurs jeux vidéo il faut accumuler des choses (Toiven et Sotamaa, 2011). Cet éclairage offre une piste de réflexion pour comprendre l'attrait qu'éprouvent les joueurs et celui qu'exerce la collection. Les auteurs présentent aussi certaines caractéristiques du collectionneur ${ }^{3}$ venant corroborer les caractéristiques présentées par Belk, Gijseghem, Baudrillard et Benjamin.

Bien que je ne sois pas psychologue, je me suis tourné vers la psychologie des collectionneurs pour creuser les motivations et la réflexion à l'œuvre derrière ces comportements passionnés parfois difficiles à saisir pour les non-initiés. L'apport d'Hubert Van Gijseghem est central dans mon évaluation des collectionneurs. Le choix de cette perspective permet, je crois, d'observer les comportements de ces collectionneurs et d'en distinguer des traits proprement québécois.

\section{Le ludovidéophile}

Avec l'aide des auteurs cités précédemment, et à partir de données recueillies directement dans la communauté québécoise, nous pouvons observer le collectionneur québécois de jeux vidéo sous l'éclairage de la collection classique et confirmer qu'il répond à la définition d'Hubert Van Gijseghem, ce qui permet ensuite d'analyser les traits qui lui appartiennent en propre. En m'inspirant de Gijseghem quand il traite du bibliophile, qui occupe selon lui une place bien particulière parmi les collectionneurs, $\mathrm{j}$ 'en suis arrivé au terme de ludovidéophile pour désigner le collectionneur de jeux vidéo. Dans le cas du bibliophile, Gijseghem insiste sur la nécessité que cette personne «qui aime le livre » ne s'entoure pas des objets collectionnés pour son travail ; encore une fois, sa collection ne doit pas être utile (Gijseghem, 2017, p. 70-71). Comme le bibliophile, le ludovidéophile se distingue de l'amateur possédant une grande quantité de jeux auxquels il joue, ou encore du journaliste et chroniqueur qui en accumulera beaucoup au cours de sa carrière.

On reconnait rapidement un collectionneur à sa quête constante de nouveaux objets, regroupés sous un même thème. Dans les communautés de collectionneurs, les membres qualifient souvent cette poursuite de «chasse » pour signifier l'intensité de l'activité, la comparant à un sport de traque. L'attrait de la trouvaille devient une poursuite, une aventure et peut même à elle seule transformer un voyage banal en une excitante épopée susceptible d'enrichir la collection de nouvelles pièces uniques et en parfaite condition. Bien que les collectionneurs aspirent à la plus grande qualité pour leurs objets fétiches, il n'est pas rare qu'ils choisissent une copie abimée parce que c'est l'acquisition et sa place dans la collection qui priment. Les collectionneurs soulignent toutefois la possibilité de restaurer certains de leurs objets ou encore de s'en procurer de nouveaux, 
moins usés, qui viendront prendre la place des premiers (Gijseghem, 2014, p. 122). Du côté du jeu vidéo, la restauration sera parfois la seule avenue possible puisque la détérioration des données numériques, ou bit rot, rendra les différents supports (disques magnétiques, mémoire RAM ou disques optiques) graduellement illisibles, donc impropres au jeu (Monnens, 2009, p. 140-142). Il reste préférable d'acquérir d'abord et d'améliorer ensuite. ${ }^{4}$ Ces habitudes sont fréquentes chez les collectionneurs de jeux vidéo, que l'on peut souvent observer en train de se procurer une copie de meilleure qualité tout en cherchant à vendre ou à échanger la pièce déjà possédée. Il y a aussi ceux et celles qui recherchent activement des pièces endommagées dans le but de leur redonner leur éclat original.

Une enquête (que j'ai menée en vue d'une étude qui sera présentée plus loin) indique que plusieurs ludovidéophiles, après l'achat d'articles en vue de bonifier leur collection, cherchent à vendre certaines de leurs pièces. ${ }^{5}$ Cependant, si on en croit Gijseghem, cette pratique est problématique, car elle va à l'encontre d'un des traits caractéristiques du collectionneur : le rejet du profit. L'investissement chez le collectionneur n'est jamais motivé par l'appât du gain, sans quoi l'infâme étiquette de marchand ou de revendeur lui sera accolée. Ce comportement est souvent décrié dans la communauté, où on qualifie ces individus de «faux collectionneurs » parce qu'ils s'intéresseraient davantage à un éventuel profit qu'à l'objet, à son histoire ou à sa préservation (Lafferty, Matulich et Xiao Liu, 2014, p. 2).

Si investir convient mal au collectionneur, il va sans dire que la vente est surveillée de près. Un individu à la tendance mercantile sera pointé du doigt et qualifié de vendeur, ce qui est plutôt péjoratif. Malgré cette réticence, on observe dans la communauté des ludovidéophiles que la vente y est tout de même omniprésente. Toutefois, comme pour l'acquisition d'objets de qualité inférieure, la vente trouve généralement sa source dans l'amélioration de la collection plutôt que dans la prospérité financière. Par ailleurs, en chassant les aubaines, un collectionneur pourra acheter un lot de plusieurs objets dans le but de n'en conserver que quelques-uns. Il cherchera ainsi à vendre les pièces en trop, et l'argent gagné sera réinjecté dans la collection. Il n'en demeure pas moins que l'aspect financier n'est jamais complètement écarté, dans les cercles de collectionneurs. D'une part, il permet de souligner l'avancement d'une collection et l'expertise de son propriétaire, tout en offrant un point de comparaison avec d'autres collectionneurs (Belk, 1995, p. 80). Au fil des acquisitions, plutôt que de reposer simplement sur le nombre d'objets qui la constituent, l'importance de la collection devient fonction d'une mesure plus précise et significative : la valeur marchande. D'autre part, connaitre la valeur de sa collection peut également servir à se déculpabiliser, à justifier en quelque sorte son passe-temps : si le coût est moins élevé que la valeur actuelle, il s'agirait d'une bonne affaire pouvant légitimer les nombreux achats. En mettant en regard les sommes dépensées et la valeur marchande de l'objet, le propriétaire peut exhiber son flair aiguisé pour les aubaines.

Le prestige atteint par une collection impressionnante confère ce que Mia Consalvo nomme le capital vidéoludique: "It's being knowledgeable about game releases and secrets, and passing that information on to others. It's having opinions about which game magazines are better and the best sites for walkthroughs on Internet» (Consalvo, 2009, p. 18). D'un point de vue ludovidéophilique, nous pouvons bonifier la définition de Consalvo en y ajoutant tous les savoirs connexes : connaissance de la fluctuation des prix, détection des contrefaçons, aptitudes en réparation et en restauration, connaissance de l'histoire du jeu vidéo. Par la présentation de sa 
collection au gré des discussions en ligne, un collectionneur accumule ce capital qui lui confère une forme de notoriété aux yeux de la communauté qui, ultimement, décide de ce qui accorde ou non ce capital tant convoité (Consalvo, 2009, p. 84).

Cette notoriété donne plus de poids aux avis de certains membres de la communauté quand s'engagent des discussions parfois très animées. On y assiste à des débats qui cherchent par exemple à déterminer ce qu'est un vrai collectionneur ou s'il est acceptable de jouer avec ses jeux. Pour Gijseghem, le vrai collectionneur « accumule des choses qui, apparemment, ne lui servent à rien, même si dans un passé plus ou moins lointain elles ont déjà eu une utilité objective ou instrumentale » (Gijseghem, 2014, p. 43), c'est-à-dire que l'objet n'est jamais utilisé. Or dans le cas des ludovidéophiles, ce sont ceux et celles qui ne jouent pas avec leurs jeux qui se voient juger par le reste de la communauté. Selon certains collectionneurs puristes, ce serait tout à fait inutile de simplement les exposer dans une bibliothèque : "Games are for playing " (NeoGAF, 2016), "Let them breathe! " (NeoGAF, 2016), " The games don't do anyone any good if they aren't used»(Sega-16, 2009). On retrouve ce type de commentaires dans une multitude de conversations en ligne à propos de la ludovidéophilie. On va même jusqu'à suggérer d'investir dans quelque chose de plus sérieux, comme la Bourse, et à remettre en question les intentions du collectionneur : " Go invest in some stocks and play your games » (NeoGAF, 2016), "Are you a gamer or a shelf collector?»(GameFAQs, 2017). La même réflexion se présente chez les ludovidéophiles québécois : « Il me semble que le but des jeux c'est d'y jouer » (CCJVQ, 2017a), «Collectionner pour une question de valeur ce n'est pas “collectionner" » (CCJVQ, 2017a), «Un jeu c'est fait pour être ouvert » (CCJVQ, 2017b). Nous verrons plus loin que, même quand ils sont questionnés sur leurs intentions, ils hésitent à se qualifier de collectionneurs, préférant d'abord s'afficher comme joueurs.

Comme nous l'avons vu, Catherine Carrey se démarque d'autres chercheurs en nuançant cette question de l'utilisation de l'objet de collection. Pour elle, une pièce ne perd pas automatiquement son statut lorsqu'elle est utilisée. C'est l'ajout à la collection qui doit primer, y jouer ou non étant secondaire (Carrey, 2008, p. 338). Par exemple, pour un non-collectionneur, il n'y a pas d'intérêt à se procurer plusieurs consoles Nintendo 64 de couleurs différentes, puisqu'une seule suffit pour profiter de sa ludothèque. En revanche, pour le ludovidéophile, chaque console additionnelle vient rajouter à la série bien définie qui comprend toutes les variations de ladite Nintendo 64. La capacité de chaque nouvelle machine à lire des jeux n'est évidemment pas le but premier de l'acquisition, bien que son utilisation soit possible et n'entache pas la collection. Pour les ludovidéophiles, vu la quantité impressionnante de pièces possédées, il relève de l'évidence que, même s'ils vont généralement utiliser leurs jeux et leurs consoles ${ }^{6}$, ils cherchent d'abord à ajouter des pièces précises à un ensemble défini.

Cet objectif d'ajouter des objets bien précis à sa collection marque le point le plus important de la définition du collectionneur avancée par les auteurs déjà cités : la sérialisation. On entend ici que les collectionneurs rassemblent leurs objets de prédilection selon une série de semblables dont le principe peut être évident (tous les jeux de la série Final Fantasy) ou arbitraire (les jeux préférés de son enfance). Le cas le plus extrême, couramment appelé full set, est celui de la collection comprenant tous les titres publiés sur une console donnée. 
C'est à partir de ces points jugés essentiels par Gijseghem, et corroborés par plusieurs chercheurs, que nous pouvons caractériser le ludovidéophile comme collectionneur tout en soulignant ses idiosyncrasies, le cas échéant. À la lumière de ces observations, j'en arrive à la conclusion que la collection ne représente qu'un trait de caractère possible, parmi tant d'autres, chez un passionné de jeux vidéo. Certains vont jouer davantage et s'identifier à un joueur assidu - souvent nommé hardcore gamer -, d'autres cherchent à acquérir rapidement le plus d'objets possible tout en vendant les doublons qui s'accumulent, frôlant l'étiquette de marchand ou de vendeur. Je propose donc de conceptualiser le passionné du jeu vidéo à l'image des éditeurs de personnages que l'on trouve dans les jeux de rôle classiques, où l'on crée son personnage en distribuant des points d'aptitude parmi un certain nombre d'attributs comme la force, la dextérité et l'intelligence. Le ludovidéophile sera alors défini par la prédominance d'un attribut par rapport aux autres :

Le ludovidéophile fait partie d'une communauté de passionnés de l'univers vidéoludique où chaque individu approche le jeu d'une façon qui lui est propre. Ces individus s'affirment par la prédominance de certains caractères parmi les caractéristiques communes suivantes : l'acquisition, l'organisation, la sérialisation, la transaction, l'utilisation, la socialisation et la polycollection. Ainsi, le collectionneur éprouve un puissant désir d'acquérir et d'organiser une quantité d'objets (jeux, périphériques et consoles) semblables en une série prédéfinie (réelle ou inventée). Des transactions procurant un profit peuvent être effectuées dans la mesure où elles sont secondaires par rapport à la recherche d'objets. L'utilisation des objets de collection est fréquente chez ce collectionneur. Cependant, la contribution d'une acquisition à la complétion d'une série demeure plus importante, rendant l'activité inutile aux yeux des non-initiés. De plus, il est possible, et même probable, que plusieurs types d'objets complètement distincts soient recherchés, fait qui révèle un polycollectionneur. L'acquisition, l'organisation et, surtout, la sérialisation sont de ce fait les caractéristiques prédominantes chez le ludovidéophile.

Assez générale pour rassembler plusieurs niveaux de collectionneurs, en plus de s'intégrer dans une typologie plus vaste des passionnés de jeu vidéo basée sur leurs comportements et leurs habitudes, cette « feuille de personnage » (de jeu de rôle) sert bien l'analyse du collectionneur québécois et des implications de sa situation culturelle et géographique.

\section{Portrait québécois - la méthodologie}

Pour peindre ce passionné québécois de jeux, j'ai compilé des données provenant d'une enquête réalisée dans le cadre d'une étude sur les collectionneurs et les effets de leur activité sur la préservation du jeu vidéo. ${ }^{7}$ Le noyau de l'étude consiste en un questionnaire de 33 questions visant d'abord à bien décrire le ludovidéophile moyen en plus de l'interroger afin de vérifier s'il s'intègre aisément dans les définitions classiques du collectionneur. Outre l'âge et le sexe, le questionnaire demande au répondant le nombre d'objets que compte sa collection, les types d'objets recherchés, si la vente active compte parmi ses activités, si la fréquentation de communautés axées sur le jeu vidéo compte parmi ses activités, etc. ${ }^{8}$

Comme le but était de connaitre ces individus et leurs comportements, le questionnaire a d'abord été distribué en ligne à trois communautés actives sur Facebook. Mon statut de collectionneur 
m'offre un accès privilégié aux membres de ces groupes qui affluent sur cette plateforme pour discuter et échanger. Facebook m'apparait une source de qualité pour entrer en contact avec des collectionneurs sérieux du Québec et observer les échanges culturels, sociaux et même économiques auxquels ils s'adonnent. L'observation de ces regroupements de passionnés ajoutée à plusieurs interactions au cours de nombreuses discussions a donc contribué à l'élaboration du portrait du ludovidéophile québécois.

Parmi les groupes populaires et actifs, mon regard s'est d'abord posé sur le Club des collectionneurs de jeux vidéo du Québec (CCJVQ), qui existe depuis 1999 - initialement en tant que forum de discussion - et qui ne cesse d'accroitre la population de sa page Facebook. Avec plus de 4200 membres, le Club regroupe des collectionneurs d'un peu partout au Québec mais dont une bonne proportion sont à Montréal. Le groupe Distribution aux collectionneurs (DAC) a lui aussi répondu au questionnaire. Au moment de l'étude, le groupe comptait plus de 200 membres avant d'être finalement absorbé par le CCJVQ (parce que les discussions et la population devenaient de plus en plus homogènes). Pendant son court règne, cette page a néanmoins attiré plusieurs amateurs, et son contenu se distinguait malgré tout de celui du CCJVQ. La troisième et dernière source s'éloigne des frontières de Montréal aussi bien que de la collection. Il s'agit d'une communauté de 1300 joueurs passionnés (ou hardcore gamers) au cœur de l'Abitibi-Témiscamingue : Rouyn-Noranda Gaming (RNG). En tout, 69 membres parmi les trois groupes ${ }^{9}$ ont répondu à l'appel en retournant un questionnaire. ${ }^{10}$ En plus de la collecte de données au moyen d'un questionnaire, j'ai consulté plusieurs pages de conversations menées au sein de ces trois regroupements, mais aussi des commentaires provenant d'autres plateformes en ligne - sites Web, forums de discussion, etc. - afin de mieux observer les comportements sans interaction directe de ma part. Je me suis aussi rendu à une réunion en personne où les membres du CCJVQ se rencontrent pour acheter, vendre ou échanger des jeux et autres objets de collection, en plus de profiter de l'occasion pour discuter de leur passion commune. Ces différentes sources nous offrent donc un portrait fidèle du ludovidéophile québécois.

\section{Se définir comme collectionneur : le cas de la communauté québécoise}

Une première manière d'aborder les collectionneurs du Québec consiste à étudier la description de leur passion ainsi que leurs motivations. Quelqu'un parmi les membres de la page Facebook du CCJVQ s'est demandé s'il était nécessaire de posséder un nombre exact de pièces avant de pouvoir se proclamer collectionneur ou si l'amour des objets était suffisant. La pléiade de réponses qui a suivi signale la variabilité de la définition au cœur même de la communauté. Pour un autre, ces deux caractéristiques (un grand nombre d'objets, l'amour de ces derniers) sont totalement inséparables. Un autre affirme ne pas porter attention à la taille de sa collection, affirmant posséder plus de 500 jeux sans pour autant se considérer comme un collectionneur. Selon un autre membre, collectionner consisterait à amasser des jeux auxquels on n'a pas l'intention de jouer, ce qui rappelle la perte de l'utilisation normale de l'objet dans les définitions de plusieurs chercheurs. Mais l'absence d'utilisation ne fait visiblement pas l'unanimité puisque, selon certains, la possibilité de jouer fait partie intégrante de l'expérience de la collection. Ainsi, quelqu'un confie garder des jeux particulièrement appréciés pour le cas où il désirerait y jouer un jour, donnant même le moment de sa retraite comme motivation. 
D'autres membres du Club des collectionneurs de jeux vidéo du Québec préfèrent l'aspect sélectif et archivistique à la nécessité de posséder un nombre précis d'objets. Les pièces de collection doivent être rassemblées selon une ligne directrice claire, que ce soit «pour la valeur documentaire, esthétique, pour leur prix, leur rareté, etc. » (CCJVQ, 2019). Un collectionneur plutôt actif dans le groupe, que nous nommerons Louis ${ }^{11}$, fait aussi la distinction entre les concepts de joueur, de marchand et de collectionneur : « si ta raison principale [de collectionner], c'est pas de garder, mais de revendre... T'es un revendeur [marchand]. Si ta raison principale est d'y jouer, t'es un joueur » (CCJVQ, 2019). Il fait reposer sa distinction sur la priorité de l'individu, soulignant l'existence de différents types d'amateurs de jeux vidéo au Québec. Du même souffle, on en déduit que parmi cette communauté, les collectionneurs québécois montrent une certaine difficulté à se définir. La réflexion amorcée sur la page du Club conduit un membre à lui-même noter le flou entourant ces réponses, qu'il s'explique par le côté personnel de l'acte; on « accumule » avant tout pour soi. Il retourne rapidement à une caractéristique plus classique en insistant ensuite sur le fait qu'un minimum de deux objets est nécessaire (CCJVQ, 2019). Ces témoignages ont contribué à l'élaboration de la définition présentée précédemment, laquelle met l'accent sur la priorité du ludovidéophile et ses comportements tout en prenant en considération les habitudes rappelant les grandes théories du collectionnement.

Les réponses au questionnaire distribué nous apprennent que plusieurs ludovidéophiles québécois refusent le titre de collectionneur, et ce, malgré le nombre impressionnant de jeux et autres objets que compte leur collection. En réponse à la question « Vous considérez-vous davantage comme un collectionneur ou comme un joueur? ", la moitié se sont qualifiés de joueurs avant tout, un peu plus du quart se sont dits collectionneurs et les autres ont dit se situer à mi-chemin. Alors même qu'il y a présence de plusieurs traits inhérents au collectionneur, le refus de ce rôle fait écho, entre autres, à l'étude de Toiven et Sotamaa sur les jeux distribués sur des supports virtuels (2010, p. 29). Dans cette étude, on apprend que plusieurs joueurs assidus préfèrent les supports tangibles aux supports dématérialisés. De ce fait, ils ont tendance à amasser une grande quantité de jeux et de périphériques, rivalisant parfois avec des collectionneurs avoués. Or, même si plusieurs signes distincts associés à la collection sont présents chez ces derniers, la plupart ne se considèrent pas comme des collectionneurs (ibid.).

Une fois que l'on a départagé les ludovidéophiles des joueurs passionnés, la distinction entre vrais et faux collectionneurs s'invite aussi dans les discussions lorsqu'on cherche à évaluer un autre membre. La différence que fait Louis entre collectionneur, marchand et joueur fait écho à Lafferty, Matulich et Liu qui évoquent ce qualificatif de «faux collectionneur » appliqué à l'individu qui s'intéresse davantage au profit qu'à l'objet, à son histoire ou à sa préservation (2014, p. 2). Cette distinction apparait également chez Gijseghem dans La psychologie du collectionneur : "J'ai observé $[\ldots]$ que ceux qui se nomment de «vrais » collectionneurs tiennent beaucoup à cette distinction, et quelquefois parlent avec mépris de ceux qui ne font que ramasser ou accumuler » (2014, p. 127). La frontière devient parfois mince au sein même de la communauté des collectionneurs quand vient le temps de décider de l'adéquation du titre.

Il y a d'ailleurs une sorte de sérieux appliqué à ces pratiques ludiques. Même si un joueur possède une grande quantité de jeux, une attention particulière est nécessaire pour traverser cette ligne de démarcation entre joueur et collectionneur. Charles en fait la remarque dans une conversation sur la page Facebook du Club des collectionneurs de jeux vidéo du Québec en spécifiant que « quand 
t'es rendu têteux sur les coins de boîtes pis les graffignes sur les stickers pour voir si tu fais un upgrade, t'es rendu avec la maladie du collectionneur »(CCJVQ, 2017c). Autrement dit, le ludovidéophile est quelqu'un de pointilleux jusqu'à inspecter les coins d'une boîte pour vérifier s'ils correspondent à ses normes de qualité ou si l'objet devra être changé pour une version encore plus près de la perfection. C'est ce degré d'attention qui mène Charles à qualifier le collectionnement de maladie, « une très jolie maladie ». Il n'est pas seul à faire cette comparaison, Belk souligne que plusieurs collectionneurs utilisent un vocabulaire médical pour décrire leur comportement et le comparer à une dépendance (Belk, 1995, p. 80).

Les chercheurs Coavoux et Gerber notent un comportement semblable dans le monde du jeu de table. En effet, dans une entrevue pour l'article « Les pratiques ludiques des adultes entre affinités électives et sociabilités familiales », Mireille mentionne qu'elle joue à des jeux de société en famille, mais qu'elle n'est toutefois pas du même niveau que son mari, un « vrai joueur » (2016, p. 145). Les auteurs citent aussi Michel qui admet avoir plusieurs façons de jouer, dont une où il joue à des jeux "pour joueurs », c'est-à-dire qui seraient de "vrais » jeux, plus complexes (Coavoux et Gerber, 2016, p. 148). On comprend que chez ces passionnés il existe une sorte de hiérarchie qui vient discriminer entre ce qui serait un vrai joueur ou non, tout comme chez les ludovidéophiles.

De ce fait, le « vrai » collectionneur demeure un concept flou, qui semble lié aux rapports avec les objets et à l'investissement de l'individu. Trop se tourner vers le commerce ou encore jouer davantage que faire l'acquisition de nouveaux objets semble affecter la perception des autres collectionneurs de la communauté. Ce type de critique se retrouve dans la plupart des groupes en ligne, que ce soit sur des forums de discussion ou sur les réseaux sociaux.

\section{Collectionneur distinct ou non?}

Afin d'en arriver à une conclusion sur la présence ou non de caractéristiques et de comportements propres au ludovidéophile québécois, mon analyse adoptera un plan en trois parties. Mais d'abord, nous pouvons dresser un portrait général du ludovidéophile québécois à l'aide des données que j'ai recueillies à même la communauté. D'abord, comme dans les statistiques sur la collection classique, on apprend que dans près de $90 \%$ des cas, ce sont des hommes qui collectionnent des jeux vidéo. Cette faible représentation des femmes demeure en phase avec les recherches de Gijseghem qui notait la même disparité il y a cinq ans (2014, p. 103). On ne peut imputer cette disparité au médium puisque, selon la Entertainment Software Association (ESA), la distribution des genres chez les joueurs en 2018 s'approche de la parité avec $55 \%$ d'hommes et $45 \%$ de femmes (2019). Ensuite, à partir de mon investigation, on observe que le ludovidéophile québécois est âgé majoritairement de 25 à 30 ans, légèrement en dessous de la moyenne de 34 ans (ESA, 2019). Étonnamment, la plupart des collectionneurs sondés ne s'adonnent à ce passe-temps que depuis dix ans, avec une tendance plus marquée au cours des cinq dernières. Pour $6 \%$ des répondants, la passion de la collection dure depuis plus de vingt-et-un ans. Sans que leur collection ait alors porté nécessairement sur le jeu vidéo, une majorité de répondants $(67 \%)$ auraient collectionné dans leur enfance. Quant à la taille des collections, elle varie beaucoup : $30 \%$ des ludovidéophiles possèdent de 100 à 199 pièces, et $9 \%$ possèdent plus de 1000 objets. Difficile de désigner les plus grands collectionneurs avec certitude, aucun palmarès n'existant pour les 
répertorier; au Club des collectionneurs, on estime que plusieurs restent discrets sur la taille de leur trésor pour éviter les vols.

Nous pouvons poser l'hypothèse que le gain en popularité des jeux vidéo dits rétro a amené beaucoup de nouveaux adeptes plus jeunes qui vont parfois aller jusqu'à collectionner des plateformes et des jeux plus vieux qu'eux. On comprend dès lors que, comme la montée en notoriété de la ludovidéophilie québécoise est relativement récente, on trouve un nombre moins élevé de « collectionneurs de longue date », ceux qui ont beaucoup d'expérience dans le domaine. En comparaison, les groupes américains ou internationaux comme AtariAge et NintendoAge, qui existent depuis plus longtemps, comptent un nombre d'amateurs beaucoup plus élevé, allant de 32000 à 42000 . À partir de ces caractéristiques générales, nous pouvons observer si les ludovidéophiles québécois se démarquent sous l'un ou l'autre des trois rapports suivants: économique, culturel et social.

\section{Dimension économique}

Dans mon étude, à la question « Est-ce que les jeux sont une sorte d'investissement pour vous ? », une majorité de participants répondent par l'affirmative $(55 \%)$. Le résultat est quasi identique lorsqu'ils sont questionnés sur la vente de jeux, de plateformes ou d'accessoires (56\%). Le marché étant relativement de petite taille, la revente au Québec a du mal à rivaliser avec un marché mondialisé propulsé par des plateformes comme eBay. On remarque d'ailleurs que $93 \%$ des répondants affirment utiliser des sites de vente en ligne pour se procurer leurs objets. La forte présence de vendeurs et de ressources utilisant le dollar américain influe sur le marché au Québec, spécialement quand le dollar canadien est faible. Le site Price Charting (2019) répertorie les ventes de jeux vidéo en ligne pour en calculer la valeur marchande moyenne. Ces prix en dollars américains sont ensuite convertis en vue des ventes privées dans la Belle Province sans pour autant qu'il soit tenu compte de la demande dans cette région beaucoup plus petite. Le marché américain et la demande sur Internet influencent donc forcément les transactions au Québec, poussant des collectionneurs toujours à la recherche de meilleurs prix à faire leurs achats au-delà des frontières.

Même dans des cas où des boutiques indépendantes sont accessibles, la décision d'acheter local ne fait pas l'unanimité, certains collectionneurs voulant encourager les commerces de proximité alors que d'autres donnent priorité à la possibilité de faire des économies. Les réponses obtenues récemment au Club des collectionneurs, toujours très actif, illustrent bien la diversité des avis sur le sujet : "Tout est trop cher en boutique de toute façon »; "J'achète de partout sans préférence, mais si le prix en boutique plus les taxes est plus grand qu'eBay c'est non »; "Les magasins rétro n'ont pas vraiment changé mes habitudes. C'est une source de plus, mais c'est loin d'être la seule » (CCJVQ, 2020). Pour d'autres, l'opportunité de se présenter en personne dans des boutiques spécialisées ne doit pas être négligée : «J'achète beaucoup de boutiques, surtout RetroMTL ${ }^{12}$, et de particuliers sur les différents groupes Facebook »; "Je vais acheter de l'usagé chez Souffle dans cassette ${ }^{13}$ à l'occasion quand il met la main sur de belles choses »(CCJVQ, 2020). Les commerces locaux et spécialisés comme RetroMTL et Souffle dans cassette ne sont donc pas repoussés du revers de la main, mais semblent inclus de facto dans une routine de chasse aux objets de collection. L'omniprésence d'eBay dans l'évaluation des prix et dans les transactions, en plus de la facilité d'accès, rend toutefois son utilisation plus alléchante, et c'est la même chose pour les 
multiples groupes et sites spécialisés. Le ludovidéophile québécois ne cherche donc pas forcément à faire ses transactions sur le marché local quand il lui faut acquérir un objet précis.

\section{Dimension culturelle}

Dans la mesure où une collection gravite autour des objets, nous pouvons prendre cette voie et observer le choix des pièces pour nous aider à mettre le doigt sur des traits propres aux collectionneurs québécois. La caractéristique de la langue pourrait présenter une piste fertile étant donné la position unique du Québec, qui est entouré du Canada anglais et bordé au sud par les États-Unis. Bien qu'il soit maintenant commun d'avoir accès à une traduction française d'un jeu - le studio Sabotage offre même une traduction typiquement québécoise de The Messenger (2018) — , ce luxe se faisait rare à l'époque où Sega et Nintendo dominaient le marché du jeu vidéo en Amérique. On trouve notamment, sur la Nintendo Entertainment System, une version francophone du célèbre Kirby's Adventure (HAL Laboratory, 1993). Sur la Super Nintendo, c'est dans leur langue maternelle que les joueurs québécois pouvaient sauver le monde d'Hyrule dans The Legend of Zelda : A Link to the Past (Nintendo, 1992) ou encore gagner la coupe Stanley dans NHL Stanley Cup (Sculptured Software, 1993). L'émission Montréal ce soir du 9 novembre 1993 a même consacré un moment à NHL Stanley Cup ${ }^{14}$, soulignant entre autres sa traduction française. Ces versions visant directement le marché québécois ont évidemment eu droit à un plus faible tirage que celles publiées sur le reste du continent. Généralement, des pièces plus rares comme celles-là vont réussir à attirer davantage l'attention des collectionneurs désireux d'ajouter des artefacts prisés à leur butin pour les exposer et profiter du capital vidéoludique que confèrent de tels objets. Une pareille frénésie n'est pas exclusive au Québec : en témoignent des titres hautement recherchés par la communauté internationale comme Little Samson (Taito, 1992) ou Stadium Event (Bandai America, 1987). Le premier a vu sa valeur exploser avec la popularité de la ludovidéophilie, passant d'une valeur d'environ 60 \$ en 2008 à un sommet de 1250 \$ en 2016 (Price Charting, 2019). Stadium Event s'est quant à lui vendu en juillet 2017 pour la modique somme de 41977 \$ US (Kohler, 2017).

Toutefois, ces jeux plus rares n'attirent pas particulièrement l'attention des collectionneurs québécois. En effet, qu'il s'agisse du prix de vente ou de la demande, il se trouve que les versions en langue française se démarquent à peine de leurs versions originales anglophones. Quant au prix, la différence n'est généralement que de quelques dollars, pas suffisante pour faire tourner davantage les têtes. Pour certains, comme Martin, il ne s'agit que d'une valeur ajoutée et qui n'est pas forcément recherchée. Même son de cloche pour David au sujet de Zelda : A Link to the Past, qui aimerait certes l'avoir dans sa collection en version complète mais ne fait pas d'efforts particuliers pour l'obtenir. Au sujet de Kirby's Adventure, comme David vise la série complète de la Nintendo Entertainment System, « [il veut] les deux, car techniquement la ROM est différente et [il] aime collectionner les alternatives ». Outre ces deux cas qui représentent un ajout concret à sa collection et à l'atteinte de ses objectifs, David précise qu'il joue toujours en anglais et qu'au final, ces versions canadiennes-françaises n'attisent pas sa convoitise. C'est donc l'objet qui prime, même lorsqu'un autre serait plus près du collectionneur culturellement parlant. Il est d'ailleurs intéressant de constater que certains collectionneurs s'intéressent aux jeux japonais, que ce soit pour des copies moins dispendieuses, pour les objets plus rares ou encore pour des œuvres n'ayant jamais quitté le Japon. 
Nous pouvons en conclure que, à l'opposé des collections institutionnelles comme celle du LUDOV $^{15}$, les collectionneurs privés ne s'intéressent pas particulièrement à l'aspect culturel québécois que l'on peut retrouver dans notre histoire du jeu vidéo. C'est l'objet et son ajout à la collection qui comptent. Ce rapport trouve une équivalence entre la communauté de ludovidéophiles québécois et celle des développeurs indépendants. D'abord, malgré les plus gros studios comme Ubisoft ou Electronic Arts qui ont pignon sur rue à Montréal, à Québec ou dans d'autres grandes villes canadiennes, le fait qu'ils appartiennent à des corporations internationales vient brouiller les pistes quand vient le temps de déterminer si ces productions sont québécoises ou américaines (Parker et Jenson, 2017, p. 871). Du côté des plus petits studios indépendants, le sentiment d'attachement se présente aussi de façon plus globale que locale. En effet, dans leur article intitule «Canadian Indie Games Between the Global and the Local», Felan Parker et Jennifer Jenson notent que la communauté canadienne du jeu indépendant est ouverte à n'importe qui et de n'importe où. Un développeur de la Nouvelle-Écosse affirme s'identifier d'abord aux autres studios indépendants, alors la géographie ou le lieu d'origine ne change rien (2017, p. 873). C'est la passion pour la création d'une œuvre vidéoludique qui compte avant tout. Nous pouvons tracer un parallèle avec les communautés internationales de ludovidéophiles comme NintendoAge, qui accueillent à bras ouverts tous les collectionneurs en quête de discussion et de partage, peu importe leur origine.

Toutefois, Parker et Jenson font mention de certains rassemblements locaux comme la Société ludique du Mont-Royal, à Montréal, ou la Toronto's Hand Eye Society, qui demeurent importants dans la construction de la communauté des développeurs et qui encouragent la collaboration et le partage de ressources. L'attachement n'est alors pas national, comme c'est le cas dans le monde de la musique ou du cinéma, mais local ou encore communautaire. À mon sens, ces rassemblements cimentent davantage un sentiment d'appartenance à la communauté qu'un engouement pour la création jeux vidéo représentatifs de la culture québécoise. Cette idée d'un rapprochement entre des individus qui partagent une même passion (la création ou la collection de jeux vidéo) me parait un terreau plus fertile pour saisir un caractère plus proprement québécois chez le ludovidéophile. En effet, ne serait-il pas plus adéquat de penser que le ludovidéophile québécois se définit davantage par ses relations avec ses semblables que par un quelconque attrait culturel émanant des objets qu'il accumule et dispose soigneusement dans sa collection ? C'est à cette question que l'évaluation d'une troisième et dernière dimension tente de répondre.

\section{Dimension sociale}

Finalement, en observant bien cette communauté de passionnés de jeux vidéo, et surtout quand on en fait soi-même partie, on s'aperçoit que la proximité qu'offre un territoire relativement petit comme le Québec permet de tisser des liens serrés et de faire s'intensifier et s'étendre un sentiment d'appartenance autour de cette passion commune plutôt que de sa culture. La grande majorité des ludovidéophiles affirment être membres d'un ou de plusieurs groupes de collectionneurs de jeux ${ }^{16}$ et près de la moitié y partagent des photos ou des vidéos de leur collection. ${ }^{17} \mathrm{Et}$ bien que certaines transactions aient lieu à partir de ces groupes et que les images partagées soient propices à la comparaison, plus de $80 \%$ des collectionneurs sondés ont dit ne pas se sentir pour autant en compétition avec leurs collègues lorsque je les ai questionnés à ce sujet. 
Outre le Club des collectionneurs de jeux vidéo du Québec, qui reste un endroit idéal pour discuter de leur engouement pour les jeux vidéo de toutes les époques ou pour exposer leur collection et s'informer sur les fluctuations inévitables de ce marché volatil, les ludovidéophiles du Québec fréquentent une grande variété de communautés qu'ils n'ont pas hésité à énumérer. Parmi ces regroupements de passionnés, plusieurs visent directement les amateurs québécois ou francophones (Rétrogaming Québec, ArcadeMTL, Retrogamer.ca, Québec Vintage Computer, Marché du rétrogaming). Parmi la soixantaine de participants à mon étude, on mentionne aussi Classic Game Collectors Canada comme groupe national en plus des forums et de sites Web plus généraux. Les membres de ces communautés abordent un vaste éventail de sujets, comme la valeur de certains jeux, la vente et les échanges; ils ont des discussions enflammées à propos de leurs jeux favoris ou de leurs mascottes préférées ; ils évoquent leurs souvenirs ; et certains tirent plaisir et fierté à présenter au reste du groupe leur salle de jeux bien organisée.

Depuis quelques années, « le CCJVQ organise une réunion trimestrielle où les membres peuvent se rencontrer afin de vendre, acheter, échanger et discuter » (CCJVQ, 2019). Ces réunions, qui se tiennent généralement à Montréal - où la majorité des membres résident -, témoignent de ce sentiment d'appartenance et de complicité tout en encourageant les rencontres. On y croise un bon nombre de collectionneurs qui se sont déplacés avec une impressionnante quantité d'objets qu'ils désirent vendre ou échanger avec d'autres qui pour leur part veulent renflouer leur collection ou se procurer une pièce qu'ils possèdent déjà, mais qui va remplacer celle en moins bonne condition. On y trouve aussi toutes sortes de produits dérivés connexes : des figurines ou des t-shirts, pour ne rien dire de l'artisanat inspiré par les franchises populaires. On peut par exemple se procurer des dessins d'artistes talentueux qui mettent en images nos jeux favoris, des images pixelisées construites avec des billes de plastique, des montages en trois dimensions de différentes scènes iconiques, ou encore un coussin sur lequel est imprimée une manette de Nintendo.

Ces rencontres regroupant un grand nombre d'acheteurs et de vendeurs sont idéales pour dénicher des aubaines et ajouter des pièces à sa collection à moindre coût. L'aspect communautaire refait surface de par la facilité à obtenir des rabais en discutant avec d'autres membres. Il est d'ailleurs fréquent pour les membres de la page Facebook du CCJVQ d'annoncer, avant une réunion, les jeux qu'ils souhaitent vendre ou cherchent à acquérir, ce qui ne manque pas d'encourager les échanges. Ici, le marché mondial dominé par eBay et le dollar américain fait place à un marché local et communautaire moins strict, spécialement quand vient le temps pour le vendeur de reprendre le métro ou l'autobus avec le moins de boîtes possible. Alors que l'influence américaine demeure présente quand vient le temps d'établir les prix à afficher, l'ombre de nos voisins du Sud se dissipe rapidement au fil des rencontres et des discussions. Il n'est en outre pas rare d'apercevoir un attroupement devant une table où la conversation ne roule plus sur la valeur marchande d'un article en vente, mais bien sur le développement tumultueux d'un jeu complètement différent.

En plus de la vente et de l'échange d'objets de collection, ces rendez-vous sont l'occasion rêvée pour rencontrer, en personne, d'autres amateurs côtoyés auparavant par l'intermédiaire du CCJVQ. Certaines discussions peuvent même se poursuivre en ligne, où d'autres membres viendront fournir leur expertise sur le sujet. Au fil de sa participation à la vie de la communauté québécoise des collectionneurs, un membre accumule un capital vidéoludique important qui s'additionnera à celui déjà acquis grâce à sa propre collection. Ainsi, certains individus peuvent atteindre une sorte de reconnaissance dans le cercle des initiés, devenant une référence à propos de certains pans de 
l'histoire du jeu, par exemple. À la manière de la scène locale du jeu indépendant décrite par Parker et Jenson, le ludovidéophile québécois m'apparait accorder une grande importance à la construction et au maintien d'une communauté saine, formée par des passionnés et des connaisseurs qui peuvent discuter, s'entraider et s'encourager, alors que chacun est en quête d'un objet dont il souhaite qu'il vienne s'ajouter bientôt à sa collection.

\section{La communauté tricotée serré}

On l'a vu, le collectionneur québécois de jeux vidéo peut difficilement faire fi de l'influence du marché international et de ses relations avec la massive communauté en ligne. L'offre, la demande et les prix sont fixés par cette grande population de passionnés, d'amateurs, de marchands et d'investisseurs voulant une part de la tarte. L'omniprésence d'eBay dans le marché du jeu vidéo d'occasion surpasse l'attrait pour ces curiosités qui demeureront toutefois des trésors pour un petit nombre. Même dans les quelques cas où une version d'un jeu a été spécialement conçue pour le marché canadien-français, la différence n'est pas assez marquée pour inciter une majorité de collectionneurs à en faire une cible prisée en raison d'une saveur propre à notre culture.

Malgré tout, une sous-communauté existe bel et bien : les ludovidéophiles du Québec peuvent échanger en français sur leur passion, et ce, non seulement en ligne mais aussi en personne. Sans être complètement différents de leurs confrère et consœurs du Sud et du reste du pays, ces joueurs aguerris - ils se considèrent majoritairement comme joueurs plutôt que comme des collectionneurs malgré leurs comportements caractéristiques du collectionnement - tirent leur épingle du jeu en tissant entre eux le sentiment d'une appartenance étroite. L'entraide, le partage et le plaisir demeurent centraux dans les innombrables conversations tenues en ligne, au sein de groupes comme le Club des collectionneurs, ou en personne à l'occasion des rencontres du Club maintenant devenues célèbres, chacune constituant un événement en soi. Le ludovidéophile québécois est bel et bien un collectionneur passionné, qui a en haute estime les objets collectionnés, et tout autant sa communauté. Si bien qu'un mouvement communautaire local facilite les échanges humains, encourage la coopération et favorise le partage de connaissances et d'informations. Il suffit de participer à un des événements du Club des collectionneurs de jeux vidéo du Québec pour observer comment les trois dimensions abordées (économique, culturelle et sociale) s'entremêlent pour faire ressortir un sentiment communautaire fort. Sans ces regroupements de passionnés, la ludovidéophilie québécoise risquerait de se diluer dans des échanges de plus en plus mondialisés sur le Web.

\section{Remerciements}

Nous tenons à remercier les deux évaluateurs anonymes de notre texte ainsi que Odette Provost et Bernard Perron pour leurs généreux et pertinents commentaires.

\section{Références}

Baudrillard, J. (1978). Le système des objets. Paris: Gallimard.

Barwick, J., Dearnlet, J., \& Muir, A. (2011). Playing Games With Cultural Heritage: A Comparative Case Study Analysis of the Current Status of Digital Game Preservation. In Games and Culture, 6(4), 373-390. 
Belk, R. W. (1995). Collecting in a Consumer Society. New York: Belknap Press.

Benjamin, W., \& Tiedemann, R. (2002). The Arcade Project. Cambridge, MA: Harvard University Press.

Carey, C. (2008). Modeling Collecting Behavior: The Role of Set Completion. Journal of Economic Psychology, 29(3). 336-347.

Club des Collectionneurs de Jeux Vidéo du Québec (CCJVQ). (2020). En ligne : https://www.facebook.com/groups/ccjvq/permalink/10158554428055330.

Club des Collectionneurs de Jeux Vidéo du Québec (CCJVQ). (n.d.). About. En ligne : https://www.facebook.com/groups/ccjvq/about/.

Club des Collectionneurs de Jeux Vidéo du Québec (CCJVQ). (2017). En ligne : https:/www.facebook.com/groups/ccjvq/permalink/10157571853175330/.

Club des Collectionneurs de Jeux Vidéo du Québec (CCJVQ). (2017a). En ligne : https://www.facebook.com/groups/ccjvq/permalink/10154945080770330/.

Club des Collectionneurs de Jeux Vidéo du Québec (CCJVQ). (2017b). En ligne : https://www.facebook.com/groups/ccjvq/permalink/10155159712000330/.

Club des Collectionneurs de Jeux Vidéo du Québec (CCJVQ). (2017c). En ligne : https:/www.facebook.com/groups/ccjvq/permalink/10155497167260330/.

Coavoux, S., \& Gerber, D. (2016). Les pratiques ludiques des adultes entre affinités électives et sociabilités familiales. Sociologie. 7(2), 133-152.

Consalvo, M. (2009). Cheating : Gaining Advantage in Videogames. Cambridge, MA: MIT Press.

Entertainment Software Association. (2019). 2019 Essential Facts About the Computer and Video Game Industry. En ligne : http://www.theesa.com/about-esa/essential-facts-computervideo-game-industry/

GameFAQs. (2017). Opening sealed games - yay or nay?. En ligne : https://gamefaqs.gamespot.com/boards/204-classic-gaming/74590537.

Gijseghem, H. V. (2014). La psychologie du collectionneur : essai de typologie. Longueuil, QC : GroupEditions.

Lafferty, B. A., Matulich, E., \& Xiao Liu, M. (2014). Exploring Worldwide Collecting Consumption Behaviors. In Journal of International Business and Cultural Studies, 8(1).

Kohler, C. (19 juillet 2017). Rare NES Game Stadium Events Sells For Nearly \$42,000. En ligne: http://kotaku.com/rare-nes-game-stadium-events-sells-for-nearly-42-000-1797061312.

Lowood, H. (Dir). (2009). Before It's Too Late. In American Journal of Play. 2(2), 139-166.

Neogaf. (2016). Not opening sealed games in your collection. En ligne : https://www.neogaf.com/threads/not-opening-sealed-games-in-your-collection.1277247/. 
Newman, J. (2012). Best Before : Videogames, Supersession and Obsolescence. Londres: Routledge, 2012.

Parker, F., \& Jenson, J. (2017). Canadian Indie Games Between the Global and the Local. In Canadian Journal of Communication. 42(5), 867-891.

Pearce, S. M. (Dir.). (1994). Interpreting Objects and Collections. Londres: Routledge.

Price Charting. (n.d.). En ligne : https://www.pricecharting.com

Price Charting. (n.d.). Little Samson. En ligne : https://www.pricecharting.com/game/nes/littlesamson.

Sega-16. (2009). Should I open my Factory Sealed games ?. En ligne : http://www.sega16.com/forum/showthread.php?7994-Should-I-open-my-Factory-Sealed-games.

Toiven, S., \& Sotamaa, O. (2011). Of discs, boxes and cartridges : the material life of digital games. In Proceedings from Digital Games Research Association (DiGRA) 2011, Hilversum (pp. 1-11). Hilversum, NL: DiGRA. En ligne : http://www.digra.org/wpcontent/uploads/digital-library/11312.23263.pdf.

Winget, M. A., \& Murray, C. (2009). Collecting and preserving videogames and their related materials: A review of current practice, game-related archives and research projects. In Proceedings of the American Society for Information Science and Technology. 45(1), 1-9. En ligne : https://doi.org/10.1002/meet.2008.1450450250.

${ }^{1}$ Les travaux de Susan M. Pearce ont aussi été proposés à la suite d'une première révision du texte. J'en remercie le directeur du numéro de même que l'évaluateur ou l'évaluatrice. Bien que pertinents en ce qui a trait à la collection, ils offraient du collectionement des définitions recoupant celles que nous mettions déjà à contribution. Aussi ai-je pris la décision de ne pas y recourir.

${ }^{2}$ Il me semble important ici de distinguer collection privée et collection institutionnelle puisque ultimement je m'intéresse aux collectionneurs privés, dont les collections n'embrassent pas les mêmes objectifs que les collections universitaires et muséales. Avec l'intérêt grandissant pour les études vidéoludiques, il n'est pas surprenant que plusieurs institutions se tournent vers ce médium et cherchent à se bâtir un corpus d'œuvres et d'objets significatifs (Barwick, Dearnlet et Muir, 2011, p. 376). Par exemple, le laboratoire LUDOV de l'Université de Montréal a récemment recentré l'un des objectifs de sa collection afin de donner une meilleure place aux jeux vidéo québécois. Pour un regard sur ces vastes collections, nous pouvons nous tourner notamment vers Winget et Murray (2009), Lowood (2009) ou Newman (2012).

${ }^{3}$ Il s'agit de la série, de la sélection, de l'entreposage et de l'exposition d'objets.

${ }^{4}$ Dans le jargon, on emprunte généralement à l'anglais le terme d'upgrade, qu'il s'agisse d'avoir un jeu avec une meilleure étiquette (label), d'obtenir la boîte (box) ou de disposer du livret d'instruction (instruction booklet).

${ }^{5}$ Dans mon étude, $56 \%$ des répondants affirment vendre des jeux, des plateformes ou des accessoires. Voici quelquesunes de leurs raisons : "Seulement lorsque j'ai des doubles » (répondant $\mathrm{n}^{\circ} 1$ ); «Souvent pour permettre d'acheter d'autres jeux » (répondant $\mathrm{n}^{\circ} 2$ ); «Pour me débarrasser de mes doublons » (répondant $\left.\mathrm{n}^{\circ} 11\right)$; « Doubles quand je trouve des lots» (répondant $\left.\mathrm{n}^{\circ} 13\right) ;$ « Revendre les doubles » (répondant $\mathrm{n}^{\circ} 20$ ).

${ }^{6}$ Parmi les répondants de mon étude, $97 \%$ affirment jouer avec leur collection ; c'est dire qu'ils l'utilisent.

${ }^{7}$ Une partie des résultats utilisés dans cet article provient de mes travaux sur les collectionneurs de jeux vidéo dans le cadre d'un mémoire de maîtrise intitulé Étude de l'impact des collectionneurs sur la préservation et la conservation du jeu vidéo, mémoire déposé à l'Université de Montréal en octobre 2018. En ligne: https://papyrus.bib.umontreal.ca/xmlui/handle/1866/22064?locale-attribute=fr.

${ }^{8}$ On trouvera la totalité des questions et des réponses dans l'annexe de mon mémoire de maîtrise. 
${ }^{9}$ Même si le partage du questionnaire était encouragé, presque tous les répondants appartiennent à l'un de ces trois groupes; une seule personne y aurait eu accès par l'intermédiaire d'une connaissance. Pour encourager la participation, les répondants n'avaient pas à s'identifier.

${ }^{10}$ Afin d'offrir de la flexibilité aux répondants qui ne seraient pas à l'aise avec toutes les questions (par exemple de divulguer l'ampleur de leur collection), il n'était pas obligatoire de répondre à chacune des questions. Pour cette raison, le nombre de réponses par questions demeure, à mon avis, acceptable : de 60 à 69.

${ }^{11}$ Les noms utilisés pour attribuer les commentaires — prélevés dans les conversations en ligne — aux collectionneurs sont fictifs. Le numéro d'ordre des répondants renvoie quant à lui aux réponses de mon questionnaire.

${ }_{12}^{12}$ RetroMTL est une boutique de jeux vidéo qui a pignon sur rue à Montréal : https://retromtl.com/.

13 Souffle dans cassette est une boutique en ligne spécialisée en jeux vidéo rétro : https://www.souffledanscassette.com/fr/.

14 Archives de Radio-Canada: https://ici.radio-canada.ca/nouvelle/1058083/super-nintendo-console-snes-minihockey-archives.

15 « Le LUDOV possède une bonne collection de jeux (près de 5000 jeux à ce jour). Mais en dehors de regroupements de jeux pour les cours de nos programmes, nous n'avons jamais pensé les acquisitions en termes de séries ou de corpus particuliers. Cependant, des discussions et un partenariat avec la Guilde du jeu vidéo du Québec nous ont fait réaliser qu'il serait important d'axer une part de notre travail de préservation sur les jeux créés au Québec. Nous allons donc attribuer une partie de nos budgets à l'acquisition de jeux vidéo créés au Québec. Nous sommes à élaborer les grandes lignes de ce projet et espérons lui donner une visibilité particulière sur le site du LUDOV dans un avenir rapproché » (Bernard Perron et Francis Lavigne, responsable et coordonnateur de LUDOV, correspondance personnelle).

${ }^{16}$ Réponse à la question « Fréquentez-vous une communauté de collectionneurs ? ».

${ }^{17}$ Réponse à la question « Partagez-vous des images (photos ou vidéos) de votre collection sur des pages Web dédiées aux collectionneurs?». 